\title{
Leap Motion evaluation for assessment of upper limbs motor skills in Parkinson's Disease*
}

\author{
A. H. Butt, E. Rovini, C. Dolciotti, P. Bongioanni, G. De Petris, F. Cavallo
}

\begin{abstract}
The main goal of this study is to investigate the Leap Motion Controller (LMC) potential for the objective assessment of motor dysfunctioning in Patients with Parkinson's disease (PwPD). The most characteristic clinical signs in Parkinson's Disease (PD), such as slowness of movements, frequency variation, amplitude variation, and speed, were extracted from the recorded LMC data. Data were clinically quantified by using LMC software development kit (SDK). In this study, 16 PwPD and 12 control healthy subjects were involved. A neurologist assessed the subjects during the task execution, assigning them a score according to the MDS/UPDRS-Section III items. Features of motor performance from both subject groups (patients and healthy controls) were extracted with dedicated algorithms. Furthermore, in order to find out the significance of such features from the clinical point of view, machine learning based methods were used. Overall, our findings showed the moderate potential of LMC to extract the motor performance of PwPD.
\end{abstract}

\section{INTRODUCTION}

Parkinson's Disease (PD) is a growing neurological disorder in the aging society. The most common characteristic clinical signs are tremor, muscular rigidity, postural instability, bradykinesia and hypokinesia, caused by a loss of brain dopaminergic neurons [1]. About $70 \%$ of PwPD experience tremor in the early disease stage: therefore, the tremor measurement is a significant parameter for diagnosing and continuously monitoring Patients with Parkinson's disease (PwPD) [2]. According to the Parkinson's Disease Foundation, one million Americans are living with PD, and approximately 60,000 Americans are diagnosed with PD each year. Similarly, 1.2 million Europeans suffer from it and this number is forecasted to double by 2030 [3]. To monitor the disease progression repeated clinical examinations are required, and this leads to high logistic costs and also affects the patients' quality of life. On the same time, visual examination of motor tasks based on semi-quantitative rating scales, such as the Hoehn-Yahr (HY) Scale [4] and the motor Section of the Movement Disorder Society/Unified Parkinson's Disease Rating Scale (MDS/UPDRS-Section III) [5], leads to high inter-rater and intra-rater variability due to neurologist subjectivity [2]. To

* Research supported by TIM, Pisa, Italy.

A. H. Butt, E. Rovini and F. Cavallo work at the BioRobotics Institute, Scuola Superiore Sant'Anna, Pontedera, (Pisa), Italy - phone: +39-0587/ 672152; fax: +39-0508/83497; e-mail: abdulhaleem.butt@santannapisa.it.

C. Dolciotti works at the Department of Translational Research of new Technologies in Medicine and Surgery, University of Pisa, Italy.

P. Bongioanni works at the Departmental Section of Severe Acquired Brain Injures- University of Pisa, Italy.

G. De Petris belongs to the TIM WHITE (Wellbeing and Health Innovative Technologies) Joint Open Lab, Pisa, Italy. date, there is no standard tool to achieve the objective assessment of PwPD on a clinical scale. The main challenge is to develop a reliable, high sensitive and fully wearable device that minimizes the discomfort of the aging people. Many studies proposed methods to automate the PwPD assessment by using wearable devices such as wireless triaxial accelerometers, triaxial gyroscopes [6,7], virtual touchpads, or electro-oculogram (EOG) signal analysis (i.e. analysis of the electric signals originating in the eye) [8]. The advantages of accelerometers include small size and relatively high sampling frequency. The main disadvantage is that they need to be attached to the fingers, which may affect their motion performance [9]. Furthermore, for each gram of additional mass a sensor adds, the peak frequency of finger tremor decreases up to $0.85 \mathrm{~Hz}$ and also affects the acceleration amplitude [10]. Recently, several studies were focused on commercially available non-contact optical devices, like Leap Motion Controller (LMC) and Microsoft Kinect sensors for the objective assessment of PwPD. Motion capture of human hand is very complex due to independent $37 \mathrm{pt}$ movements, and the commercial systems have strong 0.514 in limitations. The systems based on the three-dimensional $(3-13.1 \mathrm{~mm}$ D) infrared camera for the finger recognition or the other devices based on the camera recognition, suffer from line-ofsight obstructions and need heavy computation requirements [11]. In the recent years, depth sensors such as LMC and Microsoft Kinect sensor which provide 3-D depth data of the scene, have much potential for object segmentation and 3-D hand gesture recognition [12]. As compared to kinetic sensor and other depth sensors cameras, LMC claims to provide more detailed information about palm direction, fingertip positions, palm center position, distance between two finger tips, speed, angle and other relevant points. This information provided by LMC dedicated algorithms with no extra computational work is needed. Moreover, the precision of LMC is also higher than other depth sensors [13]. Some studies on LMC were focused on real patients to extract the different characteristics of the hand movements to assess the tremor. Recent studies showed that LMC was also used in the gaming for stroke and PD patients, particularly for upper limbs treatment. For example, the study of Hosseinpour [14] was focused on mobility therapy for PwPD by using both kinetic sensors and LMC: in such a study hand velocity was considered to monitor the PD progression. In the Chen et al.'s work [9] LMC was used to clinically quantify the essential tremor, providing algorithms to describe its amplitude. Differently, Güttler [15] aimed to monitor and detect hand tremor (essential or parkinsonian), while gesture controlling service robots novel approach was introduced. Finally, in the study of Johnson [10], Tremorometer and LMC were used in order to estimate the potential of both 
Margin requirements for the other pages

Paper size this page A4

devices to assess the resting tremor in PwPD, essential tremor subjects and healthy control subjects. Results showed that two features (peak power between 0 and $25 \mathrm{~Hz}$ and proportional power of peak between 0 and $25 \mathrm{~Hz}$ ), out of the 8 characteristics extracted for the resting tremor, were statistically different between devices for the healthy control subjects group. Overall classification between healthy and PwPD showed significant results with resting tremor characteristics extracted from LMC. The K-means cluster analysis, as a method of classification, was found to have an accuracy of $77.5 \%$, while the tuned cross-validated SVM classifier was found to have an accuracy of $85 \%$.

Based on the above studies, few characteristics of hand movements were measured to clinically quantify the tremor in PwPD. To understand the potential of LMC in a broad sense with other characteristics of the hand movements, an appropriate experimental protocol composed by 4 appropriate exercises was adopted for our study: postural tremor, hand opening/closing, thumb fore-finger tapping, and forearm pronation/supination. Such exercises, which were carefully selected since they would not affect the LMC claimed precision, are traditionally used by neurologists for visual examination of some items from the MDS/UPDRSSection III [16]. LMC was placed on the table (as shown in Fig. 1) in all exercises.

\section{INSTRUMENTS}

LMC (Fig. 1), is a commercially available non-contact optical device, $45 \mathrm{~g}$ in weight, that can detect the hand motion and position in 3-D. It consists of 3 infrared (IR) transmitters (LEDs) and 2 IR depth data capture cameras [17]. Both IR cameras are at a distance of $20 \mathrm{~mm}$ from the center of the LMC. The field of view in the hemispherical area is approximately $150^{\circ}$. The information regarding the user's hand, fingers, and gestures is captured as long as the hand is between $25 \mathrm{~mm}$ and $600 \mathrm{~mm}$ above the sensor center of the sensor. The hand position carried is relative to the center of LMC. According to the manufacturer specifications, the accuracy of LMC in spatial measurement can reach 0.01 $\mathrm{mm}$ : in previous studies, however, the error of spatial measurement in a static setup was approximately $0.2 \mathrm{~mm}$ and the average spatial error in continuous motion was $0.4 \mathrm{~mm}$ [5]. In this study average sampling rate during the data acquisition was $35 \mathrm{~Hz}$. The LMC has an attached programming interface called Leap Motion software development kit (SDK) that uses either high or medium level languages (Objective-C, $\mathrm{C}++$, Java) and allows access to depth data. From a functional perspective, the LMC software is able to provide a data stream in directly time-dependent frames, allowing calculation of hand gestures or motion above the sensor [17]. LMC is able to distinguish each finger individually through the identification algorithm which makes easy for each entity to be tracked throughout the motion flow. Positional data are provided for each object, with sub-millimetre accuracy, on each axis from the device centre. LMC software uses Windows or Mac and Linux to connect with the LMC-enabled applications. Once the LMC services are running, the leap application is connected to the LMC through the USB bus. Then, LMC-enabled applications have access to the LMC to receive data. Leap motion provides two kind of APIs to getting the data from LMC: Native interface and Web Socket Interface [15].

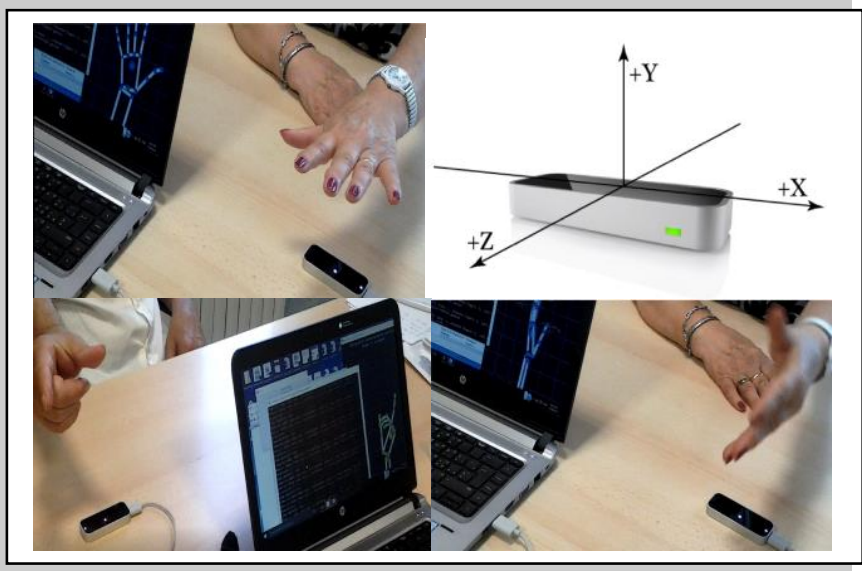

Figure 1. Coordinate system of LMC [8] and experimental setup for each exercise: postural tremor, hand opening-closing, finger tapping, forearm pronation/supination.

\section{Methodology}

In this Section, the experimental Protocol of biomechanical exercises (carried out by PwPD and healthy volunteers under the supervision of neurologists) is described in detail.

\section{A. Recruited Patients}

Sixteen PwPD (11 men, 5 women; mean age \pm SD:13.1 mm $67.9 \pm 6.9$ years old; average MDS/UPDRS-Section III scores \pm SD: $20.2 \pm 11.9$; average Hoehn and Yahr stage \pm SD: $(1.6 \pm 0.5)$ and 12 healthy controls ( 2 men, 10 women; mean \pm SD: $64.8 \pm 9.1$ years old) were asked to participate in this study. Exclusion criteria were impairments or diseases other than PD (i.e., orthopaedic or neurologic) that could affect the task performance. All subjects lived independently in the community. Every subject signed an informed consent form before the experiment.

\section{B. Experimental Protocol}

The experimental protocol for the analysis of movements of the upper limbs was composed of 4 exercises: pronation/supination of the forearms (PSUP); opening/closing of the hands (OPCL); thumb-forefinger tapping (THFF); postural tremor (POST). They respectively correspond to the tasks $3.4,3.5,3.6,3.15$ of the MDS/UPDRS-Section III [5]. Subjects were asked to sequentially perform the exercises for 3 times and for both upper limbs to complete the experimental session. In addition, every subjects made a short preliminary training to try all required movements. A neurologist assessed the subjects during the execution of the exercises, assigning them a score according to the tasks in MDS/UPDRS.

\section{Exercise Description}

- PSUP: the subject was directed to assume a sitting posture at rest and was asked to put the arm outstretched 
in front of him/herself, with the wrist stable, the hand in prone position and the fingers outstretched approximately $1 \mathrm{~cm}$ apart from each other. After $5 \mathrm{~s}$ in resting position, subject executed forearm pronationsupination movements as fast and as wide as possible for $10 \mathrm{~s}$. Then the subject was directed to keep hand in prone position and finger outstretched in resting position for $5 \mathrm{~s}$ more.

- OPCL: the subject was directed to assume a sitting posture at rest with the arm flexed at the elbow. The elbow was fixed on the table keeping the palm of the hand in front of the subject. After $5 \mathrm{~s}$ in resting position, subject alternatively opened and closed the hand as fast and as wide as possible for $10 \mathrm{~s}$ keeping the forearm and the wrist fixed. Then the subject was directed to keep the palm of the hand in front of subject in resting position for $5 \mathrm{~s}$.

- THFF: the subject was directed to assume a sitting posture at rest keeping the hand outstretch, in front of $\mathrm{him} /$ herself. In the starting position, the thumb and the forefinger were in open position. The subject remained for $5 \mathrm{~s}$ in resting position, then he/she tapped the forefinger against the thumb as quickly and as widely as possible for $10 \mathrm{~s}$. Then the subject was directed to keep the thumb and forefinger in open position for $5 \mathrm{~s}$.

- POST: the subject was directed to assume a sitting posture at rest and was asked to put the arm outstretched in front of him/herself with wrist stable, the hand in prone position and the fingers outstretched approximately $1 \mathrm{~cm}$ apart from each other. He/she remained in this position for the whole duration of the exercise for 20 seconds.

\section{Extracted Features}

For each exercise that subjects performed using the LMC, biomechanical parameters as frequency, amplitude, speed and smoothness of the movements were measured (Table I). Through the use of the LMC C++ APIs standalone program, a simple user's interface was developed in which the 4 exercises were selected in order to record the data from LMC. Furthermore the acquired data from LMC were analysed by means of appropriate algorithms implemented in Matlab ${ }^{2} R 2015 b$ to extract the features from each exercise. Since the PD tremor ranges between 5 and $12 \mathrm{~Hz}$ and essential tremor between 4 and $8 \mathrm{~Hz}$, a band-pass Butterworth filter was applied with cut-off frequency $14-\mathrm{Hz}$ to eliminate the frequency noise [15]. Since a physical guideline was not provided to PwPD to support them in correcting placing hand and fingers, some experiments were repeated, because of the uncorrected placement of the users' hands. Peak finder algorithms by Yoder [18] was used in order to count the number of movements in all exercises. Speed of these movements were recorded using the LMC APIs which allows to measure the velocity of the fingers from different directions based on the dedicated algorithms. Fast Fourier transform was implemented to transform the time domain information recorded from LMC APIs into frequency domain. Furthermore, power of each frequency component was estimated with the power spectral density function, in order to measure the frequency and amplitude variation due to the tremor in each exercise. Standard deviation (SD) of dominant frequency in time (t) segment was considered as frequency variation. Similarly, amplitude $37 \mathrm{pt}$ $\mathrm{SD}$ in $\mathrm{t}$ segments was considered as amplitude variation. For 13.14 in postural tremor, in order to estimate the tremor strength between 8 and $12 \mathrm{~Hz}$, the power of each frequency component was estimated with power spectral density function. Since each measured finger tremor was composed of several different frequencies [9], the average power of all the frequency components should provide the adequate strength of the hand tremor in the band of interest $(8-12 \mathrm{~Hz})$.

TABLE I: Biomechanical Parameters Extracted by all Exercises

\begin{tabular}{|c|c|c|}
\hline Exercises & Extracted Features & Acronyms \\
\hline PSUP & $\begin{array}{l}\text { - Number of rotational movements } \\
\text { - Supination speed } \\
\text { - Pronation speed } \\
\text { - Variability of frequency } \\
\text { - Variability of amplitude }\end{array}$ & $\begin{array}{l}\text { Num-PS } \\
\text { wps } \\
\text { wsp } \\
\text { fSD-PS } \\
\text { tetaSD-PS }\end{array}$ \\
\hline OPCL & $\begin{array}{l}\text { - Number of opening/closing movements } \\
\text { - Hand opening speed } \\
\text { - Hand closing speed } \\
\text { - Variability of frequency } \\
\text { - Variability of amplitude }\end{array}$ & $\begin{array}{l}\text { Num-OC } \\
\text { wop } \\
\text { wcl } \\
\text { fSD-OC } \\
\text { tetaSD-OC }\end{array}$ \\
\hline THFF & $\begin{array}{l}\text { - Number of thumb-forefinger tapping } \\
\text { - Opening speed } \\
\text { - Closing speed } \\
\text { - Variability of frequency } \\
\text { - Variability of amplitude }\end{array}$ & $\begin{array}{l}\text { tapTF } \\
\text { woTF } \\
\text { wcTF } \\
\text { fSD-TF } \\
\text { tetaSD-TF } \\
\end{array}$ \\
\hline POST & $\begin{array}{l}\text { - Signal strength of the movement } \\
\text { - Relative power in the band of interest of postural tremor }(8-12 \mathrm{~Hz})\end{array}$ & $\begin{array}{l}\text { PwrP } \\
\text { PwrpP2 }\end{array}$ \\
\hline
\end{tabular}




\section{EXPERIMENTAL RESULTS}

\section{A. Test-Retest Reliability with Wilcoxon signed Ranks}

Before directly focusing on the results related to motor skills, the repeatability of the device was tested. For this purpose, a non-parametric test Wilcoxon was performed with SPSS21. The Wilcoxon Signed Ranks Test was used as a two-sided test for the null hypothesis that the differences between trials come from a distribution with zero median [10]. Test failed to reject the null hypothesis except one characteristics as shown in TableII. The only null hypothesis that was rejected was the thumb fore-finger tapping closing speed (WCTF). Which directs that trail for WCTF had significant difference. In all other characteristic's acquired from the device there is no significant difference in two repeated trails in all features according to Wilcoxon analysis.

\section{B. Classification}

In order to classify the PwPD and healthy control subjects extracted features were entered in to the state of art machine learning classifier's Neural network (NN), Support vector machine (SVM) and Logistic Regression (LR). For this purpose, subjects were labeled in two classes: 0 for healthy subjects and 1 for PwPD. In order to measure the classification performance, from the classifier all the aware metrics of the diagnostic performance such as sensitivity, specificity, positive and negative predicted value, were measured. Leave-one-out cross-validation was used in SVM. The NN $70 \%$ data set was considered a training set. A level of $15 \%$ was used to validate that the network was generalizing and to stop training before overfitting. The last $15 \%$ was used as a completely independent test of network generalization with 10 hidden neurons. LR was used with tenfold cross validation. The primary concept of LR is to estimate the probability of success in the values given by the explanatory features. The logistic regression classifier is basically used to measure the relationship between dependent variables (target labels) and one or more independent variables (predictors or features) by estimating probabilities using the logistic function or sigmoidal function [19]. The results shown in the Table. 3 confusion matrix of all Classifiers LR, NN and SVM. Maximum obtained classification results from LR was $82.14 \%$ with $66.7 \%$ sensitivity and $93.8 \%$ specificity, with NN classification accuracy was $71.4 \%$ with $41.7 \%$ sensitivity and $93.8 \%$ specificity. Similarly, with SVM maximum obtained accuracy was $85.71 \%$ with sensitivity $83.5 \%$ and specificity $87.5 \%$.
C. Performance of Healthy and Parkinson Subjects.

Performance of the patients and healthy subjects in all exercises shown in the Fig.2. It can be observing in general opening movements in healthy subjects are higher than PwPD. Some PwPD showed almost same performance as healthy control. Similarly, in the case of pronation supination in general frequency of pronation supination are higher in healthy subjects as compare to PwPD. Similarly, in the postural tremor between 8 to $12 \mathrm{~Hz}$ healthy control showed very low power which is close to zero as expected. PwPD in general showed high power between 8 to $12 \mathrm{~Hz}$. In the finger tapping taping frequency per second is high in healthy control as compare to PwPD. In all exercises some PwPD showed almost same performance as healthy control. It is a matter of fact continuous treatment with medicine can improve the patient motor skills.

TABLE II: The results of the Wilcoxon Signed Ranks Test for the difference between the two trials. Highlighted $p$ value shows there is no significant different between both trials

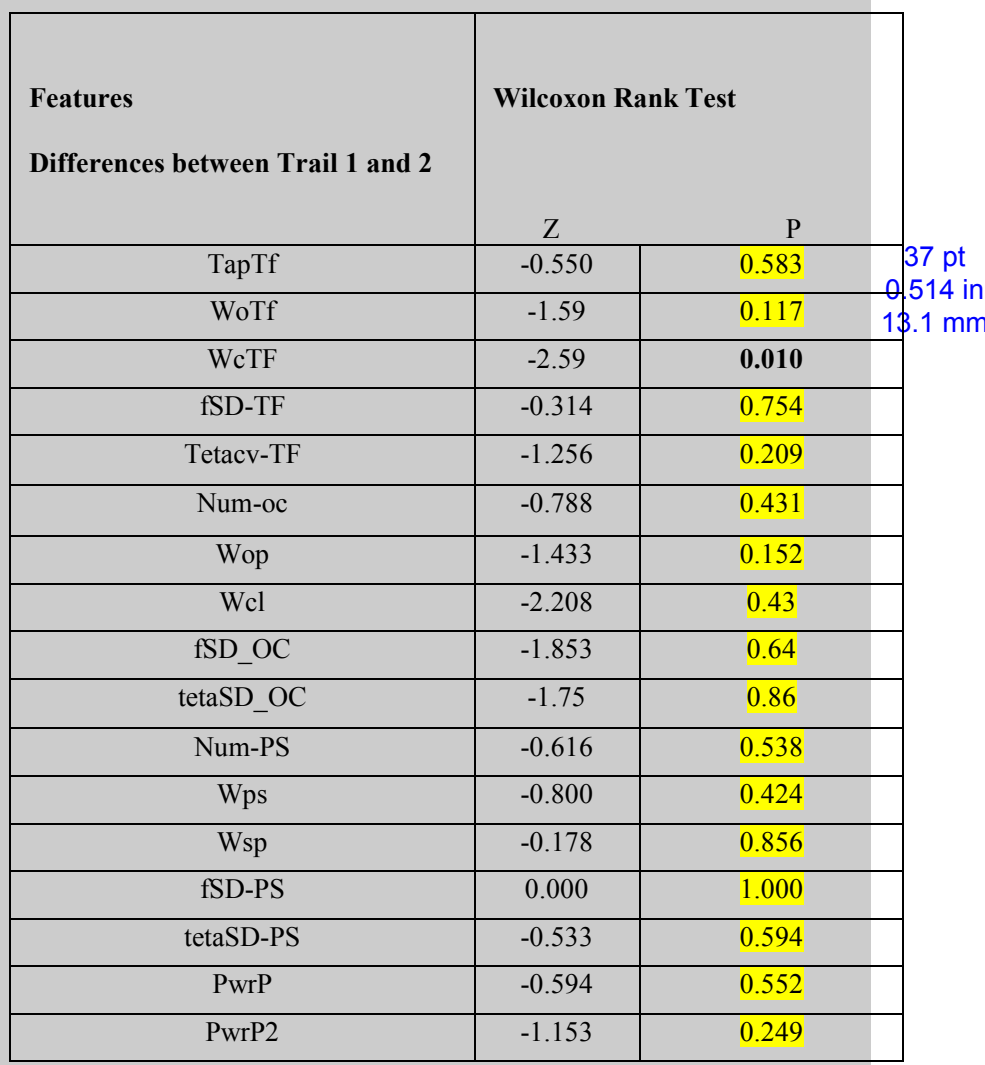


TABLE III: Classification with LR, NN and SVM between PwPD and Healthy Subjects

\begin{tabular}{|c|c|c|c|c|c|c|c|c|}
\hline \multicolumn{3}{|c|}{$\begin{array}{l}\text { LR Classification } \\
\text { Total Instances }=28 \\
\text { Correctly Classified }=23 \\
\text { Incorrectly classified }=5\end{array}$} & \multicolumn{3}{|c|}{$\begin{array}{l}\text { NN Classification } \\
\text { Total Instances }=28 \\
\text { Correctly Classified }=20 \\
\text { Incorrectly classified }=8\end{array}$} & \multicolumn{3}{|c|}{$\begin{array}{l}\text { SVM Classification } \\
\text { Total Instances=28 } \\
\text { Correctly Classified=24 } \\
\text { Incorrectly classified=4 }\end{array}$} \\
\hline & Class 0 & Class 1 & & Class 0 & Class 1 & & Class 0 & $\overline{\text { Class } 1}$ \\
\hline Class 0 & 8 & 4 & Class 0 & 5 & 1 & Class 0 & 10 & 2 \\
\hline Class 1 & 1 & 15 & Class 1 & 7 & 15 & Class 1 & 2 & 14 \\
\hline $\begin{array}{l}\text { True Positive } \\
\text { Rate }\end{array}$ & $66.7 \%$ & $93.8 \%$ & $\begin{array}{l}\text { True Positive } \\
\text { Rate }\end{array}$ & $41.7 \%$ & $93.8 \%$ & $\begin{array}{l}\text { True Positive } \\
\text { Rate }\end{array}$ & $83.3 \%$ & $87.5 \%$ \\
\hline Accuracy & $82.14 \%$ & & Accuracy & $71.4 \%$ & & Accuracy & $85.71 \%$ & \\
\hline
\end{tabular}
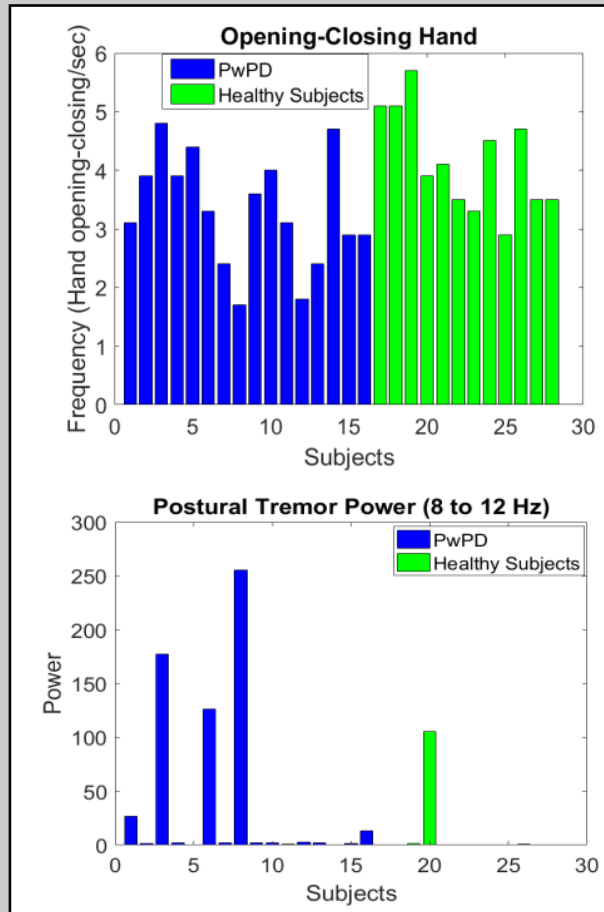
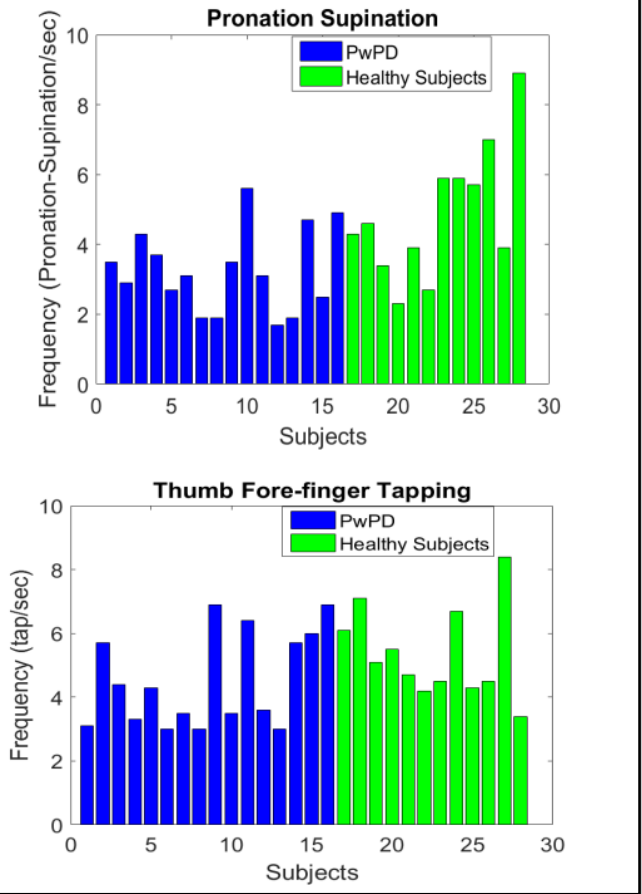

$37 \mathrm{pt}$

0.514 in $13.1 \mathrm{~mm}$

Figure2: Performance of PwPD and Control Subjects

\section{Discussion}

All the seventeen features were found to not be statistically different across trials, except one suggesting that device may be repeatable for the data acquisition for the same features. Overall the classification between PwPD and control subjects showed the moderate results. It can be observing the sensitivity and specificity in SVM is more than $80 \%$. In contrast sensitivity in $\mathrm{NN}$ is $41.7 \%$ and specificity is $93.8 \%$. Similarly, in LR sensitivity $66.7 \%$ and specificity was $93.8 \%$. Considering that PwPDs involved in this study were mainly in advanced stage of the pathology, this problem further complicated the symptom analysis because the repeated experiments caused fatigue for the patients, eventually affecting the accuracy of the measurements for symptom severity. Many of the subjects were likely taking medicine that could supress the tremor. Which would make more complicated to classify the PwPD and healthy subjects as shown in Fig.2 the performance of the both subjects. Probably, more pre-processing needed after data collection from Leap Motion to overcome these issues.

In general, working with Leap Motion is always a bit tricky when storing the big output data from the patients by the software developed in this study. On the other hand, asking a patient to do the predefined exercises in a correct way in front of the sensors avoiding the possible errors or noises is also a bit hard as well. Some exercises might also be dangerous for a group of patients having stability impairments [14]. As observed advanced patients failed to do the exercises, particularly the finger tapping task. In general, 
repeatability in the trails in all exercises direct the good ability of motion detection of LMC for PwPD.

\section{Conclusion and Future Works}

Moderate potential of LMC to assess the bradykinesia and hypokinesia observed in this work, directs the research towards the implementation of additional pre-processing algorithms to remove the noisy data and to extract the meaningful information with novel methods. Since the system was not able to correctly work on advanced PwPD, it would be beneficial to include the early stage PwPD for future investigations. Further, it would be interesting to determine if abnormal tremors in PwPD could be correctly classified out of the healthy controls with other known artificial intelligence techniques. Other supervised and unsupervised methods may provide better results. The identification and measurement of other potential features could also result to be useful, as well as the implementation of a feature selection algorithm, to identify the most significant features that could be used in the classification. Finally, it would be interesting to investigate a variety of different methods to compare the Leap Motion Controller with other wearable devices to measure the tremor in PwPD in order to study clear potential and applications of the LMC.

\section{REFERENCES}

[1] E. Rovini, "Using Wearable Sensor Systems for Objective Assessment of Parkinson's Disease", 20th IMEKO TC4 International Symposium and 18th International Workshop on ADC Modelling and Testing Research on Electric and Electronic Measurement for the Economic Upturn Benevento, Italy, September 15-17, 2014

[2] Alam, Md Nafiul, et al. "Tremor quantification of Parkinson's diseasea pilot study." International Conference on Electro Information Technology (EIT) IEEE, USA 2016.

[3] Dorsey, ER, et al, "Projected number of people with Parkinson disease in the most populous nations, 2005 through 2030." Neurology, vol. 68 no. 5, pp. 384-386, 2007

[4] M. Hoehn and M. D. Yahr, "Parkinsonism : onset, progression , and mortality," Neurology, vol. 17, no. 5, pp. 427-442, 1967.

[5] C. G. Goetz et al., "Movement disorder society-sponsored revision of the unified Parkinson's disease rating scale (MDS-UPDRS): Scale presentation and clinimetric testing results," Movement Disorders, vol. 23, no. 15, pp. 2129-2170, 2008

[6] Filippo Cavallo, et al. "Preliminary evaluation of SensHand V1 in assessing motor skills performance in Parkinson Disease" Rehabilitation Robotics (ICORR) 2013 IEEE International Conference on. IEEE, USA 2013.

[7] Rovini, Erika, et al. "Empowering Patients in Self-Management of Parkinson's Disease through Cooperative ICT Systems." Optimizing Assistive Technologies for Aging Populations (2015): 251.

[8] Dror, Ben, et al. "Automatic assessment of Parkinson's Disease from natural hands movements using 3D depth sensor", 28th Convention of Electrical \& Electronics Engineers in Israel (IEEEI) IEEE, Israel 2014.

[9] Chen, Kai-Hsiang, et al. "Development of method for quantifying essential tremor using a small optical device." Journal of neuroscience methods, vol 266, pp. 78-83, 2016

[10] Matthew J. Johnson, "Detection of Parkinson Disease Rest Tremor", M.S. thesis, Dept. Electrical Engineering and Applied Science., Washington University., USA, 2014
[11] T. Bennett, J. Wu, N. Kehtarnavaz and R. Jafari, "Inertial Measurement Unit-Based Wearable Computers for Assisted Living Applications: A signal processing perspective", IEEE Signal Process. Mag., vol. 33, no. 2, pp. 28-35, Mar. 2016.

[12] Hossein Mousavi Hondori1,"A Review on Technical and Clinical Impact of Microsoft Kinect on Physical Therapy and Rehabilitation" Hindawi Publishing Corporation Journal of Medical Engineering, vol 2014,

[13] Lu, Wei, Zheng Tong, and Jinghui Chu. "Dynamic Hand Gesture Recognition with Leap Motion Controller." IEEE Signal Processing Letters., vol 23.9 , pp. 1188-1192, 2016

[14] Nastaran Hosseinpour, "Implementation of the Kinect sensor and Leap motion for distance study of motor skills in Parkinson's disease," M. S thesis, Dept. Signal and Systems., Chalmers University., Gothenburg Sweden, 2016

[15] J. Güttler "Unobtrusive Tremor Detection and Measurement via Human-Machine Interaction" The 5th International Conference on Current and Future Trends of Information and Communication Technologies in Healthcare (ICTH)., Berlin, Germany 2015

[16] E. H. Miller, "Study and the analysis of the leap motion sensors and software development kit (SDK) for the implementation of visual human interface" Degree. Project Dept. Señales, Sistemas y Radiocomunicaciones., Escuela Técnica Superior de Ingenieros de Telecomunicación., Madrid Spain 2015

[17] Staretu, Ionel, and Catalin Moldovan. "Leap motion device used to control a real anthropomorphic gripper." International Journal of Advanced Robotic Systems., vol 13, no 3, pp. 113, 2016

[18] N. Yoder. (2013), PeakFinder - Quickly finds local maxima (peaks) or minima (valleys) in a noisy signal, [Online] Available: http://www. mathworks.com/matlabcentral/fileexchange/25500-peakfinder

[19] Musa, Abdallah Bashir. "Comparative study on classification performance between support vector machine and logistic regression." International Journal of Machine Learning and Cybernetics vol 4, no. 1 pp. 13-24, 2013 